\title{
Percepción de los alumnos sobre su primer año de universidad. Facultad de medicina Universidad de Chile
}

\author{
Students perceptions about their first year at the university. \\ University of Chile. Faculty of Medicine
}

\author{
Ilse M. López Bravo ${ }^{1}$, Zulema Vivanco Skarneo² y Enrique Mandiola Cerda ${ }^{1}$. \\ Departamento de Educación en Ciencias de la Salud. Facultad de Medicina Universidad \\ de Chile ${ }^{1}$. Facultad Ciencias de la Salud Universidad Andrés Bello
}

Se diseñó un estudio que permitiera describir la percepción de los estudiantes de las ocho escuelas de la Facultad de Medicina de la Universidad de Chile, al terminar su primer año académico, relacionada con adaptación a la vida universitaria; ambiente afectivo, calidad de la docencia, ambiente físico y cumplimiento de expectativas.

Material y método: Se diseñó un instrumento, que fue validado por expertos, con 66 aseveraciones tipo Likert. Se dio puntaje a cada respuesta. Para el análisis, la escala total se dividió en cuatro categorías.

Resultados: Respondieron 496 estudiantes. Sólo 23\% logró "adaptación a la vida universitaria" Entre los factores que la afectan: cantidad de contenidos de las asignaturas; carga horaria y falta de orientaciones prácticas de cómo estudiar.

El "ambiente afectivo" $80 \%$ lo considera "bueno". 75\% percibe "regular calidad de la docencia";

Alta proporción percibe "docentes que sólo se interesan en pasar la materia" y otros "desmotivados por la docencia".

Se encontró preferencia por clases expositivas y participativas; por los trabajos de grupo en aula, no fuera de ella; el aprendizaje a través de los trabajos de

Correspondencia:

Ilse M. López Bravo

Facultad de Medicina. Universidad de Chile

Independencia 1027. Comuna Independencia.

Santiago. Chile

Casilla 7. Correo 7. Independencia.

Telefax 56(02)9786002 ilopez@med.uchile.cl laboratorio y las guías de estudio. Las preguntas de las evaluaciones son percibidas como desconectadas de los contenidos de las clases.

Se requiere revisar actitudes de docentes que hacen que el alumno sienta que "debe caerle bien", que con frecuencia "son descalificados", que son percibidos " muy lejanos" y "menos humanos" que lo esperado.

Palabras clave: Percepción, estudiantes, primer año de universidad, transición, expectativas, facultad de medicina.

A study was designed with the aim of describing the perception of students from the eight schools in the Faculty of Medicine of the University of Chile, at the end of their first academic year, with respect to the following: adjustment to university life, emotional environment, quality of teaching, physical environment and fulfillment of expectations.

Material and method: An instrument comprising 66 Likert-type statements was designed and validated by experts. A score was given for each response. For the analysis, the total scale was divided into four categories.

Results: 496 students responded. Only $23 \%$ felt they had achieved "adjustment to university life". Among the factors affecting this were the amount of subject material, the time commitment and the lack of practical guidance regarding study techniques.

$\mathbf{8 0} \%$ considered the "emotional environment" to be "good" and 75\% regarded the "quality of teaching" as average. A high proportion felt teachers were only 
interested in teaching the content, while others were regarded as lacking motivation to teach. Students prefer lectures and participatory activities, classroom-based group work and learning through laboratory work and study guides. The evaluation questions were perceived as "unrelated to the taught content". It is necessary to review the attitudes of certain teachers who make students feel they have to behave in a particular way so as to be accepted. They often feel disqualified. Teachers are perceived as being "very distant" and "less human than expected".

Key words: perception, first year university, students, transition, medical school

\section{INTRODUCCIÓN}

En su primer año de universidad los alumnos enfrentan problemas que surgen tanto de la transición que viven como adolescentes como por lo que significa el paso de la escuela a la universidad. Inevitablemente lleva un sentido de pérdida y al mismo tiempo de nuevas oportunidades. Estas generan expectativas y sueños que se espera lograr. La forma como se equilibran las pérdidas y oportunidades y se disminuye el grado de tensión que se produce es crucial para el estudiante que ingresa a la universidad ${ }^{1}$

Existe abundante bibliografía empírica y basada en investigación para orientar a los profesores y administradores universitarios en el proceso de facilitar la adaptación del estudiante que ingresa. Sin embargo la literatura esta muy segmentada y generalmente no tiene un fundamento teórico adecuado. La mayor parte de las investigaciones se refieren a problemas específicos que ignoran la amplia variedad de influencias que actúan sobre los alumnos de primer año $0^{1-6}$

De allí entonces que el modelo conceptual para estudiar el impacto que tiene el "college" en los alumnos de primer año propuesto por los profesores Patrick T. Terenzini y Robert D. Reason del Center for the Study of Higher Education, Pennsylvania State University resulta un instrumento integrador de los diversos factores que impactan a los estudiantes de primer año $^{7}$.

El modelo consiste en tres conjuntos de influencias: la experiencia individual de cada alumno, el contexto organizacional y el ambiente estudiantil o de pares.

Los estudiantes llegan a primer año con una variedad de experiencias personales, académicas y sociales que les proporciona las estrategias básicas para enfrentar las exigencias de la vida universitaria. Estas características previas tienen una poderosa influencia en el proceso de aprendizaje en la universidad.

En el contexto organizacional el modelo incluye las estructuras internas con sus políticas y prácticas, los programas académicos y las actividades extracurriculares y la cultura del profesorado que refleja la filosofía educacional que sustenta su práctica y la forma como perciben su rol y el de los alumnos.

El tercer componente es el ambiente estudiantil o de los pares. Comprende los valores, normas, creencias, actitudes y expectativas que caracterizan al cuerpo estudiantil en su totalidad y que se ha señalado como "la fuente mas potente de influencias sobre el desarrollo estudiantil durante los años del college".

Con el propósito de indagar lo que perciben los estudiantes de las diferentes carreras del área de la salud que estudian en la Universidad de Chile se realizó un estudio en el que se plantearon los siguientes objetivos:

1. Determinar la percepción global que los estudiantes, que terminaron el año académico tienen sobre su primer año de Universidad, con relación a:

a) Su propia adaptación a las exigencias academicas

b) $\mathrm{Al}$ ambiente afectivo en relación con sus pares y docentes

c) La calidad de la docencia

d) $\mathrm{Al}$ ambiente físico en que se desarrollan las actividades

e) El cumplimiento de expectativas con respecto a la carrera

f) Sus expectativas para el año siguiente.

2. Describir la percepción del total de estudiantes encuestados según componentes de cada variable.

\section{MATERIAL Y MÉTODOS}

Se trata de un estudio descriptivo sobre la base de las respuestas a un instrumento tipo Likert, especialmente diseñado por los autores y posteriormente validado y corregido según juicio de tres expertos. En el instrumento se presentan 66 aseveraciones a las cuales el estudiante debe responder su grado de acuerdo con cinco alternativas desde: "total acuerdo", a "total desacuerdo". 
Se definieron seis variables para las cuales se planteó diferente número de aseveraciones o componentes:

a) Adaptación a la vida universitaria. Esta escala consta de 12 aseveraciones, varía entre un mínimo de 12 y un máximo de 60 puntos, con cuatro categorías de similar amplitud. Incluye percepción de carga horaria; cantidad y dificultad de contenidos; adaptación a exigencias de la carrera; rendimiento académico; grado de dificultad, disponibilidad de tiempo para vida familiar y social; alteración del sueño

b) Ambiente afectivo en relación con sus pares y docentes: con 13 aseveraciones, la escala de esta variable varía entre 13 y 65 puntos, mide actitudes favorables y desfavorables percibidas en los docentes y compañeros

c) Calidad de la docencia: Con 13 aseveraciones entre un máximo de 65 puntos y un mínimo de 13 , se exploró Metodología docente; forma y contenido de los métodos de evaluación

Ambiente físico en que se desarrollan las actividades: Se plantearon 10 aseveraciones que originaron una escala entre 10 y 50 puntos. Incluye cantidad y condiciones de salas, servicios higiénicos, salas de estar, casinos, kioscos y biblioteca. d) El cumplimiento de expectativas con respecto a la carrera: con 14 aseveraciones y la escala varió entre 14 y 70 puntos. Incluye seguridad en su elección; concordancia entre contenidos e información previa sobre la carrera; razones por las cuales se matriculó en ella

e) Expectativas para el año siguiente: 4 aseveraciones la escala varió entre 5 y 20 . Cambio de carrera, posibilidades de continuar

Para facilitar el análisis se asignó puntaje a cada respuesta. El instrumento fue aplicado al finalizar el año académico al 65,2\% de los estudiantes ingresados a primer año en 2002 a las ocho carreras que se dictan en la Facultad de Medicina de la Universidad de Chile. (Tabla 1)

\section{RESULTADOS}

ADAPTACIÓN DEL ESTUDIANTE A LAS EXIGENCIAS ACADEMICAS. (Tabla 2). La mayoría de los estudiantes percibe una "adaptación insuficiente" o "no adaptado" y Solo uno de cada cuatro logró algun nivel de adaptación a la vida universitaria.

Percepción del AMBIENTE AFECTIVO. 82,5\% de

Tabla 1: Estudiantes que respondieron el cuestionario según escuela. Facultad de Medicina. Universidad de Chile 2001.

\begin{tabular}{l|l|l|l}
\hline ESCUELA & Total ingresados & Node Respondientes & \% respondientes \\
\hline Enfermería & 109 & 40 & 36,7 \\
Fonoaudiología & 49 & 11 & 22,4 \\
Kinesiología & 54 & 45 & 83,3 \\
Medicina & 235 & 203 & 86,4 \\
Nutrición y dietética & 50 & 30 & 60,0 \\
Obstetricia & 131 & 97 & 74,0 \\
Tecnología médica & 82 & 38 & 46,3 \\
Terapia ocupacional & 51 & 32 & 62,7 \\
\hline Total & 761 & 496 & 65,2
\end{tabular}

Tabla 2: Distribución porcentual de estudiantes en cada variable, según nivel de puntajes.

Escala y mediana de puntaje en cada variable

\begin{tabular}{|c|c|c|c|c|c|}
\hline VARIABLE & $\begin{array}{l}\text { MUY ALTOS PERCEPCION } \\
\text { MUY POSITIVA }\end{array}$ & $\begin{array}{l}\text { ALTOS PERCEPCION } \\
\text { POSITIVA C/REPAROS }\end{array}$ & $\begin{array}{l}\text { BAJOS PERCEPC } \\
\text { ALGO NEGATIVA }\end{array}$ & $\begin{array}{l}\text { MUY BAJOS PERCEPCION } \\
\text { TOTALMENTE NEGATIVA }\end{array}$ & Mediana \\
\hline $\begin{array}{l}\text { ADAPTACION VIDA UNIVERSITARIA } \\
\text { (escala 12-60) }\end{array}$ & 1,0 & 22,0 & 58,2 & 18,8 & 31 \\
\hline AMBIENTE AFECTIVO (escala 13-65) & 15,0 & 67,5 & 17,1 & 0,4 & 46 \\
\hline CALIDAD DOCENCIA (escala 13-65) & 0,3 & 21,5 & 75,0 & 3,2 & 36 \\
\hline AMBIENTE FISICO (escala 10-50) & 0,0 & 15,5 & 65,0 & 19,5 & 38 \\
\hline $\begin{array}{l}\text { CUMPLE EXPECTATIVAS RESPECTO A } \\
\text { LA CARRERA (escala 14-70) }\end{array}$ & 43,9 & 43,6 & 11,3 & 1,2 & 54 \\
\hline $\begin{array}{l}\text { EXPECTATIVAS AÑO PROXIMO } \\
\text { (escala 5-20) }\end{array}$ & 84,9 & 11,7 & 2,4 & 1,0 & 17 \\
\hline
\end{tabular}


los estudiantes se ubicó en las dos categorías más favorables al proceso.

La mayoría de los estudiantes manifiestan una percepcion "algo negativa" de CALIDAD DE LA DOCENCIA

En AMBIENTE FÍSICO la percepción se concentra en las dos categorías mas negativas. 38 fue el puntaje más alto observado, $24 \%$ por debajo de lo esperado.

La gran mayoría de los estudiantes perciben que LA CARRERA LOGRA CUMPLIR SUS EXPECTATIVAS.

Casi la totalidad de estudiantes indican el propósito de continuar en la misma carrera.

DISTRIBUCIÓN DE ESTUDIANTES EN CADA COMPONENTE DE LA VARIABLE "ADAPTACION A LAS EXIGENCIAS ACADEMICAS. (Tabla 3)

Destaca la alta proporción de estudiantes que se siente agobiado por la cantidad de contenidos de algunas asignaturas; que consideran el horario de clases muy recargado; que han tenido problemas para dormir desde que entraron a la universidad; que sienten que sus obligaciones estudiantiles no les deja tiempo para compartir con su familia y amistades y quienes sienten que su rendimiento habría sido mejor si hubiesen tenido orientación de cómo estudiar. Mas de la mitad de los estudiantes siente que este año fue muy difícil para ellos.

\section{DISTRIBUCIÓN DE ESTUDIANTES EN CADA COMPONENTE DE LA VARIABLE "AMBIENTE} AFECTIVO.(Tabla 4)

Esta contiene aspectos negativos y positivos, que se refieren a su relación con los docentes y con los compañeros. La proporción de estudiantes que se muestra de acuerdo con los aspectos positivos del "ambiente afectivo", es notablemente mayor que la observada con los negativos, lo que muestra un ambiente favorable por parte de docentes y compañeros; pero hay aseveraciones que presentan entre $15 \%$

Tabla 3: Porcentaje de estudiantes en cada componente de la variable "adaptación a la vida universitaria".

\begin{tabular}{|c|c|c|c|c|c|}
\hline COMPONENTES DE VARIABLE & TOTAL ACUERDO & ACUERDO & INDIFERENTE & DESACUERDO & TOTAL DESACUERDO \\
\hline $\begin{array}{l}\text { 22. La cantidad de contenidos de algunas asignaturas } \\
\text { me hace sentir agobiado }\end{array}$ & 45.7 & 37.6 & 6.4 & 8.1 & 2.2 \\
\hline $\begin{array}{l}\text { 23. Cantidad de contenidos que entregan en algunas clases } \\
\text { son excesivos }\end{array}$ & 42.9 & 38.8 & 8.7 & 8.2 & 1.4 \\
\hline $\begin{array}{l}\text { 28. Siento que algunos estudiantes muestran poco interés } \\
\text { en aprender }\end{array}$ & 5.2 & 10.7 & 20.3 & 48.7 & 15.1 \\
\hline 31. El horario de clases está recargado & 18.3 & 33.2 & 15.9 & 29.8 & 2.8 \\
\hline 32. Me ha sido muy difícil adaptarme a las exigencias de la carrera & 12.3 & 32.8 & 11.5 & 35.8 & 7.6 \\
\hline $\begin{array}{l}\text { 33. Siento que mi rendimiento habría sido mejor si me hubieran } \\
\text { orientado cómo estudiar }\end{array}$ & 27.2 & 36.8 & 15.7 & 16.1 & 4.2 \\
\hline 38. Este primer año fue muy difícil para mí & 24.4 & 34.0 & 12.3 & 23.9 & 5.4 \\
\hline 43. Mi rendimiento es mejor con los trabajos de grupo & 16.3 & 46.1 & 18.9 & 14.9 & 3.8 \\
\hline 51. He logrado mi meta para este año & 9.7 & 29.2 & 12.1 & 39.6 & 9.4 \\
\hline $\begin{array}{l}\text { 62. Desde que entré a la Univ. tengo problemas para dormir } \\
\text { en las noches }\end{array}$ & 12.1 & 17.9 & 9.5 & 29.4 & 31.1 \\
\hline $\begin{array}{l}\text { 63. Desde que entré a la Univ. He compartido menos } \\
\text { con mi familia }\end{array}$ & 37.1 & 39.2 & 4.8 & 12.1 & 6.8 \\
\hline 64. Desde que entré a la Univ. Siento que no tengo tiempo & 30.8 & 41.3 & 6.2 & 16.7 & 5.0 \\
\hline
\end{tabular}

para compartir con mis amigos

Tabla 4: Porcentaje de estudiantes en cada componente de la variable "ambiente afectivo"

\begin{tabular}{|c|c|c|c|c|c|}
\hline COMPONENTES DE VARIABLE & TOTAL ACUERDO & ACUERDO & INDIFERENTE & DESACUERDO & TOTAL DESACUERDO \\
\hline 12. Siento que tengo que caerle bien al docente & 8.9 & 23.1 & 42.7 & 17.5 & 7.8 \\
\hline 13. Los docentes nos descalifican con mucha frecuencia & 3.0 & 17.7 & 22.9 & 42.7 & 13.7 \\
\hline 19. Siento a los docentes muy lejanos & 8.5 & 29.6 & 22.5 & 33.8 & 5.6 \\
\hline 16. Esperaba docentes más humanos & 8.7 & 17.9 & 19.7 & 40.0 & 13.7 \\
\hline $\begin{array}{l}\text { 15. Mis compañeros son indiferentes a mis dificultades } \\
\text { de aprendizaje }\end{array}$ & 3.0 & 10.7 & 15.1 & 47.0 & 24.2 \\
\hline 17. Esperaba compañeros más humanos & 6.8 & 12.9 & 16.1 & 40.5 & 23.7 \\
\hline 14. Me he sentido solo en mis dificultades de aprendizaje & 8.7 & 24.6 & 21.9 & 36.8 & 8.0 \\
\hline 11.Docentes le han ayudado a superar dificultades de aprendizaje. & 5.8 & 18.7 & 23.1 & 42.7 & 9.7 \\
\hline $\begin{array}{l}\text { 18. Los compañeros son solidarios con quienes tienen dificultades } \\
\text { de aprendizaje }\end{array}$ & 1.6 & 15.1 & 12.3 & 44.5 & 26.5 \\
\hline 20. He logrado establecer buenas relaciones con mis compañeros & 1.4 & 4.0 & 4.8 & 47.1 & 42.7 \\
\hline 21. He logrado establecer buenas relaciones con mis docentes & 0.8 & 8.7 & 21.5 & 55.7 & 13.3 \\
\hline 65. En la Universidad he encontrado muchos amigos & 2.6 & 8.3 & 8.9 & 49.1 & 31.1 \\
\hline 66. La vida universitaria me ha hecho feliz & 6.2 & 9.7 & 18.1 & 43.5 & 22. \\
\hline
\end{tabular}


Tabla 5: Con relación a la calidad de la docencia:

\begin{tabular}{|c|c|c|c|c|c|}
\hline COMPONENTES DE VARIABLE & TOTAL ACUERDO & ACUERDO & INDIFERENTE & DESACUERDO & TOTAL DESACUERDO \\
\hline 26. A la mayoría de los docentes solo le interesa pasar materia & 12.9 & 32.4 & 17.9 & 34.2 & 2.6 \\
\hline 30. Algunos docentes están desmotivados para hacer clase & 9.9 & 36.2 & 16.1 & 33.0 & 4.8 \\
\hline 34. Prefiero clase participativa & 21.1 & 40.9 & 19.5 & 15.5 & 3.0 \\
\hline 35. Prefiero clase expositiva & 14.7 & 38.9 & 18.5 & 21.9 & 6.0 \\
\hline 36. Prefiero los trabajos de grupo en sala & 17.3 & 43.1 & 18.7 & 16.5 & 4.4 \\
\hline 37. Prefiero los trabajos de grupo para desarrollar fuera de la sala & 8.5 & 19.9 & 20.3 & 35.4 & 15.9 \\
\hline $\begin{array}{l}\text { 40. Trabajos en laboratorio fueron relevantes para el aprendizaje } \\
\text { de los contenidos }\end{array}$ & 24.4 & 44.8 & 12.9 & 12.1 & 5.8 \\
\hline 41. Me agrada que el docente dicte su clase & 17.7 & 36.9 & 21.3 & 19.3 & 4.8 \\
\hline 42. Me agrada desarrollar guías de estudio & 10.9 & 38.8 & 25.0 & 18.9 & 6.4 \\
\hline 24. Las formas de evaluación son justas & 7.2 & 47.1 & 13.3 & 27.8 & 4.6 \\
\hline 25. Las evaluaciones siempre tienen alto nivel de dificultad & 18.7 & 51.5 & 11.5 & 17.3 & 1.0 \\
\hline $\begin{array}{l}\text { 27. Las preguntas de las pruebas concuerdan con los contenidos } \\
\text { tratados en clase }\end{array}$ & 3.6 & 22.9 & 9.1 & 54.9 & 9.5 \\
\hline 61. El horario de clases estuvo mal distribuido & 14.3 & 25.1 & 19.3 & 28.0 & 13.3 \\
\hline
\end{tabular}

Tabla 6: Con relación a la calidad de la docencia:

\begin{tabular}{|c|c|c|c|c|c|}
\hline COMPONENTES DE VARIABLE & TOTAL ACUERDO & ACUERDO & INDIFERENTE & DESACUERDO & TOTAL DESACUERDO \\
\hline 2. El número de salas de clases son suficientes & 14.1 & 47.3 & 6.4 & 27.4 & 4.8 \\
\hline 9. Las salas no favorecen el trabajo de pequeño grupo & 18.3 & 33.2 & 20.5 & 25.8 & 2.2 \\
\hline $\begin{array}{l}\text { 8. Debería existir espacios físicos que favorezcan trabajo } \\
\text { de pequeño grupo }\end{array}$ & 53.3 & 34.8 & 8.3 & 2.8 & 0.8 \\
\hline 7. La biblioteca tiene luz y tranquilidad suficiente para estudiar allí & 5.4 & 23.5 & 10.5 & 42.1 & 18.5 \\
\hline 6. La biblioteca tiene material suficiente & 5.6 & 25.2 & 3.6 & 50.3 & 15.3 \\
\hline 3. Opciones para alimentarse son suficientes(casinos, quioscos, etc) & 19.7 & 39.6 & 9.1 & 23.3 & 8.3 \\
\hline $\begin{array}{l}\text { 5. Los servicios higiénicos están en condiciones adecuadas } \\
\text { para el uso }\end{array}$ & 3.8 & 20.5 & 4.8 & 41.5 & 29.4 \\
\hline 4. Los servicios higiénicos son suficientes & 5.0 & 15.9 & 3.4 & 30.4 & 45.3 \\
\hline 10. Siento que se necesitan salas de estar & 59.3 & 28.0 & 9.3 & 2.8 & 0.6 \\
\hline 1. Ambiente físico favorece el estudio & 9.9 & 48.3 & 12.3 & 26.0 & 3.5 \\
\hline
\end{tabular}

y $43 \%$ de las respuestas en la categoría "indiferente" y otras que señalan que entre $20 \%$ y $30 \%$ de los estudiantes perciben una relación negativa con sus docentes y/o compañeros. Una proporción importante percibe que "La vida universitaria lo ha hecho feliz"

\section{CALIDAD DE LA DOCENCIA (Tabla 5).}

En relación a la actitud de los docentes, las percepciones son variadas en cuanto a que "solo les interesa pasar materia" o que "algunos estan desmotivados para hacer clases". Respecto al tipo de metodología se observa preferencia por la clase participativa, los trabajos de grupo en la sala de clases, el desarrollar guias de estudio y una alta proporción reconoce la importancia de los trabajos de laboratorio. En cuanto a las evaluaciones sienten que tienen alto nivel de dificultad y las preguntas no siempre concuerdan con los contenidos de las clases.

\section{PERCEPCIÓN DEL AMBIENTE FÍSICO}

(Tabla 6)

Se destaca la escasez de salas y la inexistencia de espacios que favorezcan el trabajo de pequeños grupos. Para la gran mayoría de los estudiantes la biblioteca no ofrece condiciones de tranquilidad, ilu- minación, ni cantidad de libros suficientes. Casi unánime es la percepción de necesidad de contar con salas de estar para los alumnos. Solo $58 \%$ percibe que el ambiente físico favorece el estudio.

\section{CUMPLIMIENTO DE EXPECTATIVAS (Tabla 7)}

Más del $70 \%$ de los estudiantes seleccionó su carrera de acuerdo a lo que deseaba, sienten que tienen vocación y que seran feliz con ella. Escasa frecuencia se cambiaría de carrera o "no sabe" si lo haría. Algunos perciben una diferencia entre lo que sabía sobre la carrera y lo que encontró y otros piensan que "algunas asignaturas no tienen sentido para su carrera". La mitad los jóvenes opina que la carrera debería incluir otros elementos para seleccionar a los alumnos.

\section{DISCUSIÓN Y CONCLUSIONES}

Los resultados del estudio son concordantes con los encontrados en las encuestas nacionales que se realizan en los alumnos de primer año del college en Estados Unidos, Australia y Canadá ${ }^{8-10}$

Son problemas comunes: el proceso de adaptación a la vida y cultura universitaria, las condiciones del 
Tabla 7: Cumplimiento de expectativas

\begin{tabular}{|c|c|c|c|c|c|}
\hline COMPONENTES DE VARIABLE & TOTAL ACUERDO & ACUERDO & INDIFERENTE & DESACUERDO & TOTAL DESACUERDO \\
\hline 45. Cuando me matriculé estaba seguro de lo que quería & 45.9 & 23.4 & 6.0 & 19.1 & 5.6 \\
\hline 46. Elegí esta carrera porque creo que seré feliz & 50.9 & 31.2 & 8.5 & 6.0 & 3.4 \\
\hline 47. Siento vocación por esta carrera & 53.4 & 32.8 & 7.4 & 3.8 & 2.6 \\
\hline $\begin{array}{l}\text { 55. Me matriculé en esta carrera porque no quedé en la que } \\
\text { yo quería }\end{array}$ & 11.1 & 14.9 & 4.0 & 16.1 & 53.9 \\
\hline 56. Me matriculé en esta carrera porque no quería perder el año & 5.6 & 8.5 & 5.2 & 18.1 & 62.6 \\
\hline $\begin{array}{l}\text { 53. Lo que yo sabia sobre la carrera coincide con lo que encontré } \\
\text { en la Universidad }\end{array}$ & 16.1 & 48.1 & 10.9 & 19.7 & 5.2 \\
\hline 54. Me faltó información sobre lo que es la carrera & 8.7 & 23.1 & 11.7 & 37.4 & 19.1 \\
\hline $\begin{array}{l}\text { 48. Los contenidos que vi este año no concuerdan con lo que } \\
\text { yo esperaba }\end{array}$ & 4.6 & 14.5 & 13.9 & 49.7 & 17.3 \\
\hline $\begin{array}{l}\text { 29. Algunas asignaturas de este año no tienen sentido } \\
\text { para mi carrera }\end{array}$ & 18.7 & 24.4 & 9.9 & 36.2 & 10.8 \\
\hline $\begin{array}{l}\text { 52. Este año he logrado darme cuenta lo que hace un profesional } \\
\text { de mi área }\end{array}$ & 30.8 & 46.1 & 5.6 & 13.5 & 4.0 \\
\hline 44. Si tuviera oportunidad me cambiaría de carrera & 7.7 & 7.5 & 6.6 & 21.7 & 56.5 \\
\hline $\begin{array}{l}\text { 50. La carrera debería incluir otros elementos para seleccionar } \\
\text { a sus alumnos }\end{array}$ & 19.3 & 32.8 & 23.4 & 17.1 & 7.4 \\
\hline 39. Si me hubiera organizado mejor habría tenido un rendimiento & 46.1 & 39.0 & 3.8 & 8.1 & 3.0 \\
\hline
\end{tabular}

medio físico, la relación con los profesores y sus pares, la administración del tiempo para compatibilizar las obligaciones académicas con la vida familiar y social, contar con servicios de apoyo pedagógicos, psicológicos, bibliográfico y de infotecnología, el cumplimiento con las exigencias académicas para obtener el rendimiento satisfactorio en todos los cursos.

En nuestro estudio se encontró que aproximadamente uno de cuatro alumnos podía ser clasificado como "adaptado". Como factores que dificultan la adaptación se mencionaron el número excesivo de horas semanales de clases, la cantidad de contenidos en cada asignatura.

Para la gran mayoría de los estudiantes el ambiente afectivo resulta satisfactorio, sin embargo aparecen algunos problemas relacionados con actitudes de los profesores hacia los alumnos que se consideran "descalificados" y perciben a los profesores como "muy lejanos" "menos humanos que lo esperado";"solo les interesa pasar toda la materia" y algunos no se muestran interesados en la docencia.

En cuanto a calidad de la docencia la mayoría de los estudiantes prefieren la clase participativa, sin embargo una proporción no despreciable se inclina por la clase expositiva. Se aprecia una evidente preferencia por los trabajos de grupo en aula actitud que puede asociarse a la carga horaria y a la dificultad para reunirse a trabajar fuera de horas de clase. Como actividades que favorecen el aprendizaje se mencionan las guías de estudio y los trabajos prácticos de laboratorio.

En el área de evaluación se critica la dificultad de las preguntas y su falta de concordancia con el contenido de las clases.
La mayoría de los estudiantes estima que en su primer año de universidad se cumplieron sus expectativas. Pocos piensan cambiar de carrera lo que confirma que seleccionaron la carrera que deseaban.

En relación al ambiente físico las falencias señaladas en la encuesta han sido superadas en lo que se refiere a biblioteca y al espacio para descanso y recreación. La biblioteca cuenta hoy con un moderno edificio con instalaciones que ofrecen un ambiente grato para el trabajo. Se aumentó el número de textos para cada disciplina, la suscripción a revistas científicas y la disponibilidad de computadores.

A la luz de estos resultados se le plantea a las carreras la necesidad de revisar el currículo y los programas con el objeto de disminuir la excesiva cantidad de contenidos, mejorar la calidad de la docencia en lo que se refiere a metodología, evaluación y la relación profesor-alumno

\section{BIBLIOGRAFÍA}

1. Latham Gloria., Green Pam. "The Journey to University: A study of "the First Year Experience". Faculty of Educacion, Language and Community Services. Royal Melbourne Institute of Technology.Australia.Document URL: ttp://ultibase.rmit.edu.au/Articles/dec97/greenlath1.htm. Last Updated: 19-December-1997 by Marita Mueller

2. Hargreaves, A., Earl, L.M., Ryan, J. "Scholling for change: Reinventing Education for Early Adolescents" 1996. London Falmer Press.

3. Terenzini P, et al. "The transition to college: Diverse students, diverse stories". 1994. Research in Higher Education, 35 (1): 57-73

4. Bender, Sharon. "Addressing the Issues of Social and Academic 
Integration For First Year Students". A discussion paper University of Wollongong. Australia. Ultibase, December 1997

5. Walker, James L. Ph D. "Survey of first-year University Students". University of British Columbia Edition. Prepared for The Canadian Undergraduate Consortium June 1998

6. Skipper T. "Student Development in the First College Year. A Primer for College Educators". The National Resource Center for First-Year Experience and Students in Transition" University of South Carolina. 2005/119 pages

7. Terenzini, P., Reason, R,D. "Parsing the First Year of college: a conceptual Framework for Studying College Impacts". Paper presented at the meeting of the Association for the Study of
Higher Education, Nov 19, 2005, Philadelphia, PA. Penn State Center for the Study of Higher Education.

8. Keup J, Stolzenberg E."The 2003 Your First College Year (YFCY)Survey: Exploring the Academic and Personal Experiences of College Students" 2004/94 pages

9. Krause KL, Hartley R, James R, McInnis C. "The First year Experience in Australian Universities: Findings from a Decade Of National Studies" Centre for the Study of Higher Education University of Melbourne. 2005

10. Canadian Undergraduate survey Consortium. "Survey of First-Year University students:2004. University of Victoria. 2004 